\title{
Effects of SIP in Interoperable LMR/Cellular Heterogeneous Mobile Wireless Network
}

\author{
S.Kirubakaran ${ }^{1}$, Dr.C.Manoharan ${ }^{2}$ \\ '(Assistant Professor, Department of CSE, INFO Institute of Engineering, Coimbatore, India) \\ ${ }^{2}$ (Principal, Annai Mathammal sheela Engineering College, Namakkal, India)
}

\begin{abstract}
Public safety agencies are using LMR network, which has less service availability, less multimedia services and low data rates during the emergency communication. These less services may impact the life in the critical emergency situation. The commercial cellular network provides higher service availability, more multimedia services and higher data rates. The LMR network mobile node can access the cellular network services if cellular/LMR network are interoperable. For accessing the cellular network services, the LMR network mobile node should handoff to cellular network if there is resource available. Ongoing communication may disrupt by this handoff and radio resources very intensive. We have studied Traditional SIP and Seamless SIP method with the constraints of handoff delay and resource utilization. The study presents Session Schedule Manager SIP, which achieves the optimal handoff and increase the radio resource utilization. This Paper intends optimal handoff and maximizes the resource utilization in the interoperable heterogeneous network which provides efficient communication between Disaster responder and Disaster commander.
\end{abstract}

Keywords - Hand off delay, Heterogeneous Network, Radio resource, Packet loss, SIP

\section{Introduction}

Efficient communications are crucial for disaster responders in disaster response and recovery during Disasters. The Disaster responders are requiring guaranteed reliable communication in order to save lives. Wireless mobile network is recent wireless technology which plays an increasingly important role in disaster response. Rapid Emergency response and coordinating teams are played by current Public safety LMR. The Public safety Land Mobile Radio refers to wireless systems used by public safety agencies for coordinating teams and providing rapid emergency response. Field based emergency personnel and other authorities heavily use secure, portable radio communications called Land Mobile Radios. Most public safety mobile wireless networks are based on two digital narrowband LMR technologies: APCO Project 25 and Terrestrial Trunked Radio (TETRA).APCO Project25, standardized by TIA and EIA.TETRA, standardized by ETSI. Comparisons of different LMR technology are shown in table 1. Most current LMR networks provide narrowband circuit switched digital voice service with limited support of low speed data services. In discrepancy, The 3G Cellular networks and 4G Cellular network can support Packet switched, broadband services with a variety of multimedia application that include higher data rates, voice, video and web browsing. The considerable differences in terms of services and data rates between these two kinds of wireless networks are largely due to market forces, requirements, spectrum policy and other parameters. In the interoperable Cellular/LMR networks, disaster responders can access the services in cellular networks that are not available in public safety LMR networks to increase the service availability. Besides, when a disaster responder moves out of the coverage of public safety LMR networks with an ongoing communication session, the session should be handoffed to cellular networks instead of being dropped to provide the communication continuity.

One of the most important enablers of the migration to converged IP-enabled services in a datacentric network model is session control based on the IP Multimedia Subsystem (IMS) signaling framework. This framework has been adopted by both 3GPP and 3GPP2. The IMS is designed to be access network independent, and allows interoperability for IP-enabled services across LMR/cellular networks and across different types of end user devices. It employs IETF protocols such as the Session Initial Protocol (SIP) to realize standardized reference architecture, and provides a common signaling framework for end user registration, session establishment, security, profile management, accounting, and end-to-end QoS management. It also reduces cost by allowing the ability to share session control and media resources available within the network across different SIP-based applications.

\section{Handoff Related Previous Work}

In [1], the vertical handoff decision is formulated as a fuzzy multiple attribute decision-making (MADM) problem.The two MADM ranking methods are proposed in [1]: 1) SAW and 2) TOPSIS. In [2], the network selection for vertical handoff is modeled by the analytic hierarchy process (AHP) and the GRA. The AHP decomposes the network selection problem into several sub problems and assigns a weight value to each 
subproblem. Then, the GRA is used to rank the candidate networks and selects the one with the highest ranking. In [6], we investigate the performance among SAW, TOPSIS, and GRA regarding the vertical handoff decision. In [7], the handoff decision mechanism is formulated as an optimization problem. Each candidate network is associated with a cost function, which depends on the bandwidth, delay, and power consumption. An application-oriented vertical handoff decision mechanism is proposed in [8]. In [9], a framework is proposed to compare different vertical handoff algorithms. The framework includes a path loss channel model between the mobile terminal and the access point and a Markov chain that models the user's movement between different access networks. A multilayer framework for vertical handoff is proposed in [10]. A rules engine, combined with several threshold parameters, is used to monitor the decision parameters while the handoff policies are stored in a database. The framework allows the trigger of the vertical handoff by either changes in applications, variations of the network's conditions, or preferences of the users. In [13], the vertical handoff decision is evaluated via a handoff cost function and a handoff threshold function, which can be dynamically adapted to the changes in the network environment. In [14], a vertical handoff decision algorithm based on dynamic programming is introduced. It considers the movement and location information, which is provided by the location service server.

Although there have been various vertical handoff algorithms proposed in the literature, our work is motivated by two particular aspects. First, the handoff delay needs to be taken into account during the handoff decision. Second, the radio resource utilization to be taken into account during the handoff.

\section{Effects of Handoff Delay Based on Traditional SIP}

SIP has been around for around 20 years now, and yet engineers are still finding new ways to use it to address telecommunications challenges. SIP session management addresses the challenge of operating a multivendor, multi-protocol telecommunications environment by enabling easier interconnectivity of SIP-based applications, often mediating between various proprietary implementations of SIP or SIP extensions.Session Initiation Protocol (SIP), the standards developed by IETF for initiating, modifying and terminating multimedia sessions including voice communications. SIP, a widely accepted signaling protocol, is capable of providing mobility support at the application layer, where there is the least amount of dependence on the lower layers. SIP is a simple, scalable, text based protocol that offers a number of benefits, including extensibility and provision for call/session control.

The powerful Session Initiation Protocol can be used in any application where session initiation is required; it is still too powerful to use session creation. Several wireless technical forums, e.g., 3GPP, 3GPP2, and MWIF, have agreed upon SIP as the basis of the session management of the mobile Internet. It seems that SIP will certainly be an integral part of the mobile Internet's protocol architecture. Thus, it would be desirable to use SIP to provide means of terminal, service as well as personal mobility for all applications.

The advantages of using SIP for supporting mobility are:

- Mobile users can roam across SIP environments without concern about whether they support network layer mobility or not.

- Provides a means of route optimization and improved performance for real-time services via SIP signaling messages for address binding, registration, etc.,

- Moving of a media stream from one terminal to another

The traditional SIP that provides "break-before make" scheme to reduce the handoff delay. But it does not achieve seamless handoff. There are essential request messages defined in SIP, and they are distinguished by what is called a method:

- $\quad$ REGISTER:Is used by a client to register an address with SIP server

- INVITE: Indicates that the user or services is being invited to participate in a session.

- ACK:Confirms that the client has received a final response to an INVITE request, and it only used with INVITE requests

- CANCEL:Is used to cancel a pending request

- BYE:Is sent by a user Agent Client to indicate to the server that it wishes to terminate the call

- OPTIONS:Is used to query a server about its capabilities.

In the Heterogeneous Network, traditional SIP is adopted as the communication protocol between LMR and Cellular network. In addition, Radio resource utilization is also considered in the designing of Disaster Responder Communication Network. Since the radio resource utilization is major challenges for the multimedia application. We use an example to illustrate how a mobile node(MN) handoffs from a LMR network to a Cellular network.Initially, a Mobile node with SIP URI MN@LMR in the LMR network sends an INVITE message to a RN.The RN agrees to establish a session by replying with an OK message. An ACK message is the sent back to the RN.After the setup procedure, a session is setup to exchange data. During the exchange of data between $\mathrm{MN}$ and $\mathrm{RN}$, the MN wants to handoff to the cellular network for more advanced multimedia services.

In the Traditional SIP scheme, before making the new session the old session is terminated by sending BYE message from $\mathrm{MN}$ to $\mathrm{RN}$ and $\mathrm{OK}$ message from RN to MN.The MN authenticate, authorize and 
accounting with the cellular network and IP or domain addresses assigned by DHCP as MN@cellular. After obtaining the new address, the MN sends an INVITE message to the RN via cellular network. The new session is created between MN and RN via cellular network. Now the data is exchanged between MN and RN via cellular network. In this way, the RN knows that before making new session the old session is terminated. After the handoff transaction, the MN sends REGISTER message to HSS for updating the new contact address. The Home Subscriber Server (HSS) reply the OK message after completion of update. There is no seamless handoff in this "break before make" SIP scheme.

\section{Effects of Handoff Delay Based on Seamless SIP}

The novel SIP-based seamless handoff (SSIP) scheme offers a "make-before-break" scheme to reduce the handoff delay and achieve seamless handoff. The Seamless SIP uses only four necessary messages called REGISTER, INVITE, ACK and BYE. We use an example to illustrate how a mobile node (MN) handoffs from a LMR network to a cellular network. Initially, a MN with SIP URI MN@LMR in the LMR network sends an INVITE message to a RN. The RN agrees to establish a session by replying with an OK message. An ACK message is then sent back to the RN. After the setup procedure, a session is setup to exchange data. During the session, the MN wants to handoff to the cellular network for more advanced multimedia services. It then authenticates with the cellular network and acquires IP and/or domain addresses.

We assume that the dynamic host configuration protocol (DHCP) server is used in the cellular network and the MN is assigned with an address of MN@Cellular. After obtaining the new address, the MN sends an INVITE message to the RN via the cellular network. In this way, the RN knows that the new session wants to join the ongoing session between the $\mathrm{RN}$ and $\mathrm{MN}$ via the LMR network. After negotiating the parameters, another session is established between the MN and RN via the cellular network. The RN synchronously sends data to both networks. After the handoff transaction, the MN and RN communicate through the two sessions independently and synchronously. The MN will discard any duplicate RTP packets. When the new session is setup, the MN will send a BYE message to the RN to terminate the session via the LMR network. It also updates the contact address in the HSS with the new address MN@Cellular.

Note that the data sent during this handoff period can still reach the MN due to the seamless handoff nature. Let $D_{\text {handoff }}$ be the handoff delay and $D_{A \leftrightarrow B}$ be the delay of messages transmitted between nodes $A$ and $B$. We have:

$$
D_{\text {Handoff }}=5 D_{M N \leftrightarrow R N}+2 D_{M N K \leftrightarrow C S C F}+4 D_{M N \leftrightarrow D H C H}
$$

Generally, there are three types of delay: processing delay at end nodes, transmission delay (wireless and wireline) and wireless propagation delay. The propagation delay over a wireless access network is very small and can be neglected. The delay incurred by messages involves: (a) processing delay at end nodes (MN or $\mathrm{RN})$,(b) transmission delay over wireless links, and (c) transmission delay over the Internet.

Therefore, we have:

$$
\begin{aligned}
& D_{M N \leftrightarrow R N}=D_{P, M N}+D_{T, L(D T, C)}+D_{T, I}+D_{P, R N} \\
& D_{M N \leftrightarrow C S P F / D H C P}=D_{P, M N}+D_{T, C}+D_{P, C S C F / D H C P}
\end{aligned}
$$

where $D P, M N$ and $D P, R N$ are the process delay at the $\mathrm{MN}$ and RN, respectively, DT,L, DT,C and DT,I are the transmission delay over the LMR, cellular and Internet, respectively.

Substituting (2) and (3) into (1), we have:

$$
\begin{aligned}
D_{\text {Handoff }}= & 11 D_{P, M N}+5 D_{P, R N}+2 D_{T, L}+9 D_{T, L}+5 D_{T, I} \\
& +2 D_{P, C S C F}+4 D_{P, D H C P}
\end{aligned}
$$

In addition, the Seamless SIP handoff scheme measures the radio resource utilization for the guaranteed service availability and continuity QoS.We consider an interoperable LMR and Cellular system with a single LMR cell and a single cellular cell. When first responder with an ongoing communication session moves out of the coverage of the LMR, the session can be handoff to the cellular network if resource is available.

\subsection{Algorithm:}

Step1: Session creation between MN and RN in LMR Network

Step2: Establish the voice communication between $\mathrm{MN}$ and $\mathrm{RN}$ via LMR Network Resources

Step3:

if ( $\mathrm{MN}$ moves from LMR network coverage to cellular network )

if ( Radio resource available in cellular network )

if $\left(\mathrm{P}_{\mathrm{j}} \mathrm{N}_{\leq \mathrm{TP}} \mathrm{N}_{\mathrm{N}}\right)$

Create a new session between $\mathrm{MN}$ and $\mathrm{RN}$ in cellular network 
if $\left(\mathrm{P}_{\mathrm{j}} \mathrm{H}_{\leq \mathrm{TP}} \mathrm{T}_{\mathrm{H}}^{\mathrm{H}}\right)$

MN Handoff to cellular network

Initialized multimedia communication between $\mathrm{MN}$ and $\mathrm{RN}$ via cellular network else

Dropped the communication between MN and RN in LMR network

Step5: New Call session Registration

Step6: Old Call session Termination

The new session blocking probability $P_{j}^{N}$,should be kept below a target value to guarantee the service availability. Let $T P_{j}^{N}$ denote the target value of new session blocking probability. The handoff dropping probability $P_{j}{ }^{H}$, should be kept below a target value to guarantee the service continuity. Let $T P_{j}{ }^{H}$ denote the target value of the handoff dropping probability.

\section{Effects of Handoff Delay Based on Proposed Session Schedule Manager SIP}

In order to consider Handoff delay and packet loss used SSM-SIP in heterogeneous mobile wireless network. The Radio resources are demanding to the multimedia applications. The interoperable LMR and cellular network mobile station users are using multimedia data such as voice and video for their emergency communication for the efficient disaster response. In this heterogeneous mobile wireless network, the Application server (CSCF) is used to the signaling admission point in the interconnection between cellular and public safety LMR networks. The Dynamic Host Configuration Protocol (DHCP) server is used in the cellular network and the MN is assigned with an address of MN@Cellular.The Mobile Nodes sends an INVITE message to the Remote Node in the LMR network via the cellular network. In this way, the RN knows that the new session wants to join with the ongoing session between the Remote Nodes and Mobile Nodes in the LMR network. During the MN node and RN node session process, another session will be invited. Now the nodes are waiting for the acknowledgement message. It will increase the handoff delay time and packet loss .In this situation now proposed scheme SSM-SIP (Session Schedule Manager SIP) is avoid waiting session at runtime by maintaining all sessions in virtual mode (Figure 1 and 2).The good quality of service to end users are provided and the overall radio resource are optimally utilized.

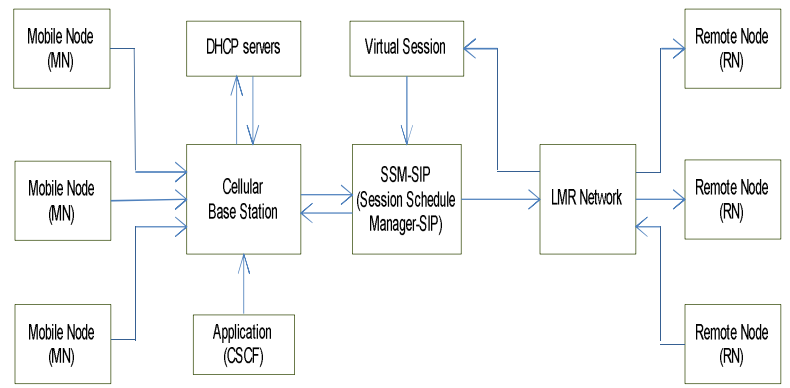

Fig. 1:SSM-SIP Architecture Diagram

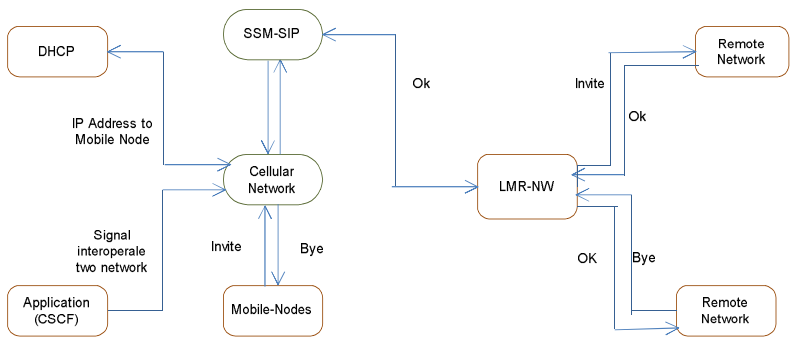

Fig. 2: SSM-SIP session scheduling

\subsection{Session Schedule Manager SIP Procedure:}

- MN sends INVITE Message to RN

- $\mathrm{RN}$ sends OK Message to MN

- MN sends ACK Message

- Intermediate solution for SSM-SIP

- Initialize Data Communication between MN and RN via LMR Network 
- MN send DHCP DISCOVER Message to DHCP server

- DHCP server send DHCP OFFER Message to MN

- MN sends DHCP REQUEST to DHCP server

- DHCP server send DHCP ACK Message to MN

- MN sends INVITE Message to RN

- $\mathrm{RN}$ sends OK Message to MN

- MN sends ACK Message to RN

- Session Blocking Probability should be $\mathrm{P}_{\mathrm{j}} \mathrm{N}_{\leq \mathrm{TP}} \mathrm{P}^{\mathrm{N}}$

- SSM-SIP create virtual sessions

- Initialize Data Communication between MN and RN via Cellular Network.

- MN sends INVITE Message to RN

- $\mathrm{RN}$ sends OK Message to MN

- MN sends ACK Message to RN, at the same time it called another new session and immediately synchronized to virtual session.

- If session finish RN sends Bye message.

\section{Results and Discussion}

The simulation scenario is designed specifically to assess the impact of network density on the performance of the protocols. The impact of network density is assessed by deploying $20-34$ nodes over a fixed square topology area of $1000 \mathrm{~m} \times 800 \mathrm{~m}$ using $250 \mathrm{~m}$ node Transmission range. Simulation scenario shows two different group of node where one group of nodes called LMR access network. Another group of nodes are cellular mobile nodes. The mobile nodes are used traffic model such as CBR (Constant Bit Rate ) and VBR (Variable Bit Rate) provide voice and video services. Figure 3 shows Simulation of heterogeneous network creation, new session creation and Mobile Node (MN) handoff.

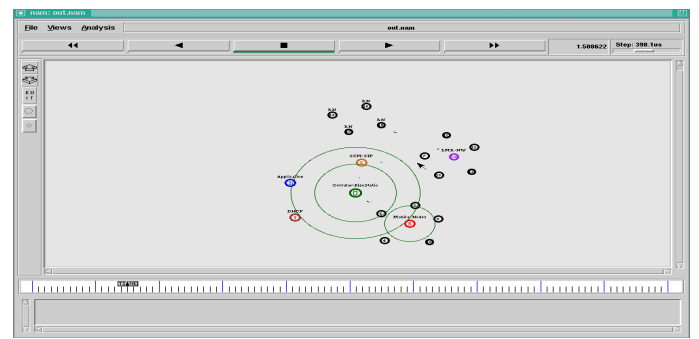

Fig.3: MN Handoff from LMR Network to Cellular Network

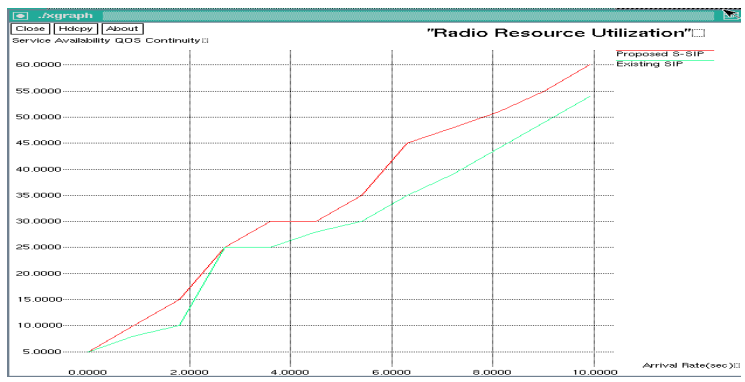

Fig.4: Comparison of Overall Radio Resource Utilization with SIP and S-SIP

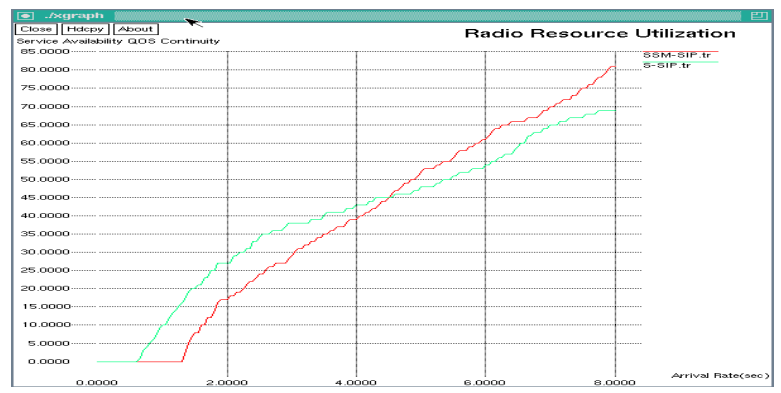

Fig. 5: Comparison of Overall Radio Resource Utilization with S-SIP and SSM-SIP 
We have compared the handoff delay in the traditional SIP with Seamless SIP. Here the handoff delay is very near to zero. Because maximum number of handoff are seamless handoff which means the handoff dropping probability and new session blocking probability are below the threshold value. The performance Interoperability in Heterogeneous Mobile Wireless Networks were measured with respect to metrics like Packet throughput ratio ,delivery ratio, end - end delay etc. Our proposed Session Schedule Manager SIP method ensures the optimal performance than the existing traditional and seamless SIP methods. In the SIP simulation report, throughput ratio is 0.61 where as seamless SIP shows 0.62.The sending and receiving packets in SIP are 770 and 766 respectively. But in the case of seamless SIP sending and receiving packets are 785 and 781 respectively.PDF of traditional SIP is 97.48 where as 99.79 PDF in Seamless SIP. Finally, average end to end delay in traditional SIP is $36.00 \mathrm{~ms}$ where as $32.09 \mathrm{~ms}$ in Seamless SIP. The Transmission delay is also compared for the traditional SIP and Seamless SIP.

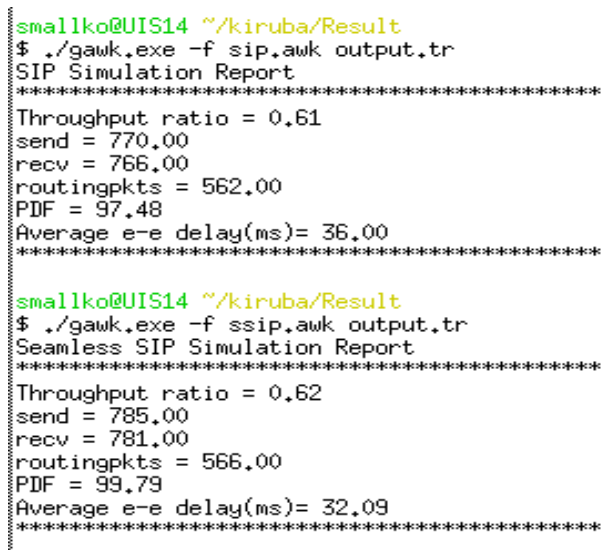

Fig.6: Traditional and Seamless SIP Simulation Report

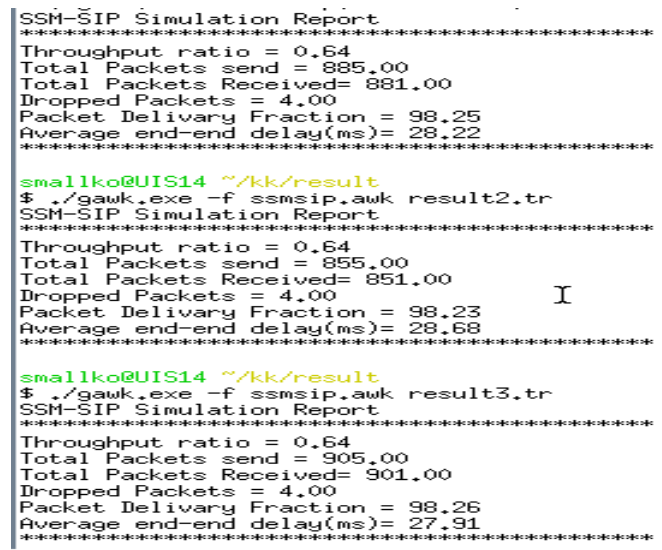

Fig.7: Traditional and Seamless SIP Simulation Report

The SSM SIP sending and receiving packets are 905 and 901 respectively. The PDF of Seamless SIP is 97.48 where as 98.26 PDF in SSM SIP. The average end to end delay in seamless SIP is $32.09 \mathrm{~ms}$ where as 27.9 $\mathrm{ms}$ in Seamless SIP. The performance of Interoperable LMR and Cellular network is increased by using the SSM SIP from the simulation results.

\section{Conclusion}

We have studied the handoff delay and packet loss in the interoperable heterogeneous mobile wireless network. The traditional SIP and Seamless SIP has been studied for the constraints of handoff delay and packet loss. In our performance study, we observed the delay incurred in traditional SIP is more than the Seamless SIP due to "break before make" scheme and "make before break" respectively. In the traditional SIP, the old session is terminated before making the new session. The session blocking probability and handoff dropping probability is above the target session blocking and handoff dropping probability. But the Seamless SIP make a new session before breaking the old session. The SSM-SIP is presented with the virtual session creation. The session blocking and handoff dropping probability of SSM-SIP in the interoperable heterogeneous mobile wireless network are below a target session blocking and handoff dropping probability. Due to this we can conclude the 
good throughput is achieved. Finally, the optimal handoff delay and packet losses are achieved and radio resource utilization is also improved by using SSM-SIP.

\section{References}

[1] W. Zhang, "Handover decision using fuzzy MADM in heterogeneous networks," in Proc. IEEE WCNC, Atlanta, GA, Mar. 2004, pp. 653-658.

[2] Q. Song and A. Jamalipour, "A network selection mechanism for next generation networks," in Proc. IEEE CC, Seoul, Korea, May 2005, pp. 1418-1422.

[3] H. Wang, R. Katz, and J. Giese, "Policy-enabled handoffs across heterogeneous wireless networks," in Proc. IEEE WMCSA, New Orleans, LA,Feb. 1999, pp. 51-60.

[4] K. Yoon and C. Hwang, Multiple Attribute Decision Making: An Introduction.Newbury Park, CA: Sage, 1995.

[5] M. R. Kibria, A. Jamalipour, and V. Mirchandani, "A location aware three-step vertical handoff scheme for 4G/B3G networks," in Proc. IEEE GLOBECOM, St. Louis, MO, Nov. 2005, pp. 2752-2756.

[6] E. Stevens-Navarro and V. Wong, "Comparison between vertical handoff decision algorithms for heterogeneous wireless networks," in Proc. IEEE VTC - Spring, Melbourne, Australia, May 2006, pp. 947-951.

[7] F. Zhu and J. MacNair, "Optimizations for vertical handoff decision algorithms," in Proc. IEEE WCNC, Atlanta, GA, Mar. 2004, pp. 867-872.

[8] W. Chen and Y. Shu, "Active application oriented vertical handoff in next generation wireless networks," in Proc. IEEE WCNC, New Orleans, LA, Mar. 2005, pp. 1383-1388.

[9] A. Zahran and B. Liang, "Performance evaluation framework for vertical handoff algorithms in heterogeneous networks," in Proc. IEEE ICC,Seoul, Korea, May 2005, pp. 173-178.

[10] A. Sur and D. Sicker, "Multi layer rules based framework for vertical handoff," in Proc. BROADNETS, Boston, MA, Oct. 2005, pp. $571-580$.

[11] C. Guo, Z. Guo, Q. Zhang, and W. Zhu, "A seamless and proactive end-to-end mobility solution for roaming across heterogeneous wireless networks," IEEE J. Sel. Areas Commun., vol. 22, no. 5, pp. 834-848,Jun. 2004.

[12] O. Ormond, J. Murphy, and G. Muntean, "Utility-based intelligent network selection in beyond 3G systems," in Proc. IEEE ICC, Istanbul, Turkey, Jun. 2006, pp. 1831-1836.

[13] A. Hassawa, N. Nasser, and H. Hassanein, "Tramcar: A context-aware cross-layer architecture for next generation heterogeneous wireless networks," in Proc. IEEE ICC, Istanbul, Turkey, Jun. 2006, pp. 240-245.

[14] J. Zhang, H. C. Chan, and V. Leung, "A location-based vertical handoff decision algorithm for heterogeneous mobile networks," in Proc. IEEE GLOBECOM, San Francisco, CA, Nov. 2006, pp. 1-5. 